\title{
Research on the Literature Sensibility in Ancient Literature Researches
}

\author{
Liyu Chen
}

Sichuan Huaxin Modern Vocational College, Chengdu Sichuan, 610000, China

\author{
Keywords: Ancient times, Literature research, Literature sensibility.
}

\begin{abstract}
Chinese ancient literature includes poem, novel, drama and so on. Thereinto, poem mainly embodies the meaning of literature and intangible of art, therefore, understanding and studying poem needs researchers' good sensibility, and analysis on the artistic image and themes of novel and drama also cannot leave author's sensibility. Therefore, sensibility and literature, basic theory have same effects in ancient literature researches, all of them are indispensable conditions to study ancient literature. This paper mainly analyzes and discusses the literature sensibility in ancient literature researches.
\end{abstract}

\section{Introduction}

Literature creation is originated from life and is the sublimation of life. Authors always connect his/her literature creation with social background and life at that time, thus to embody his/her feelings on life, ad this kind of deep feeling can be conveyed to readers though literature works, thus to realize the dialogue across time and space between the authors and readers to induce readers' emotional resonance to literature works. The writing keynotes of Chinese ancient literature creations are mainly authors' perceptions, literature works are cohesion of authors' close observation on life and perception on art. This shows that studying ancient literature works requires researchers to understand the emotions in the works thus to get into the authors' inward world and to experience the core meaning of the works.

\section{The specific function of sensibility in ancient literature researches}

In ancient literature researches, sensibility mainly refers to litterateurs' or literary critics' intuitive feelings, experience, judgment and understanding ability on the emotions, meanings, language arts and thoughts in the literature works, also includes their intuitive feelings and acute insight on literature works ${ }^{[1]}$. The perception on literature works can help readers understand the creation core of literature works, only accessing to the core of literature works can realize perception on the authors' spirit and the artistic value of the works, can help readers be melt into the created thoughts and atmospheres of the authors, to ensure the accuracy and rationality of the emotional analysis on literature works. Meanwhile, researchers' excellent sensitivity can deeply dig the historical value of literature works, thus to stimulate the development of historiography and provide references for restoring history.

\section{Influence factors on Chinese ancient literature researches}

Chinese ancient civilization history has always been on the leading position in the world civilization, its glory radiated to the culture stage of the whole world. Chinese ancient literary creation has gone through thousands of years' history, it experienced lots of historical changes within thousands of years' development process, therefore, its creation background was very complicated, this is also the reason that Chinese ancient literature researches cannot be developed deeply and comprehensively. Meanwhile, researchers on Chinese ancient literature are with different levels of quality, there are few professional researchers with high literary quality, and influenced by market economy, people are easy to be seduced by economic benefits, thus the ancient literature works with little economic profit entered the downturn. All these reasons caused the low level of current ancient literature researches, researches are hard to get substantial progress, difficulties in the researches are 
also difficult to be effectively overcame. It still needs large quantities of research inputs and needs to introduce in excellent ancient literature researchers to achieve rapid development of ancient literature researches.

The above three reasons are recognized as main influence factors on ancient literature researches, but many people neglect another obstacle factor on ancient literature researches, that is researchers' low sensitivity. Literature sensitivity is the basis and premise of ancient literature research, because ancient literature has high requirements on artistic conception, many ancient literature works contain author's deep emotion, thoughts and social backgrounds,therefore, only with high literature sensitivity can better understand the artistic conceptions and thoughts in the ancient literature works, can promote the progress of ancient literature researches. Literature work itself is an emotional description on author's spiritual journey, its ideological connotation and literary value are contained in the literary images, thus only with deep study, understanding and perception on the images, story line and languages of the literature works, can highlight the essential thoughts of the literature works. Literature historian and literary critics' research process on literature works are all started from images, after getting into the literary figures, to grasp the literature work as a whole needs to establish emotional resonance with literary figures, to help researchers experience the author's spiritual progress and reappear the work contents, all these cannot leave the function of perception ${ }^{[2]}$.

\section{Literature sensitivity is the core of the Chines ancient literature research objects}

\section{Research on ancient poems.}

In Chinese ancient literature researches, poem has always been the main study object, poem researches can be divided into presentation and representation, two kinds of art forms, while researches always place emphasis on presentation art form. Poems in this kind of presentation form become the presentation literature that pays attention to connotation and intelligence. In poem creations, ancient poets strove for inspiration and timing, as Lu Ji said in his work Lyrics that "Once creativeness comes, it is numerous, complicated and cannot be tided.”; stove for interests and appreciation, as Dong Fangshu wrote in his work Zhao Mei Zhan Yan that "Write with one's interests"; stove for perception, as Lv Benzhong said in his work that "For papers need perception, perception comes from hard work not by luck." These ancient sayings were all embodiment of ancient literature intelligence, inspiration of literature creation is not ever-lasting, but an instant feeling, it can be understood as the instantly break out of the authors' thoughts, though this kind of creative thought happens in a moment and goes quickly, but the emotion, content and thoughts in these literature works that created under this kind of inspiration are profound. Therefore, evaluation of literature works always abide by the art appreciation principles of abundant meanings, this made the ancient poem development always set conception and charms as the main creative connotation, pastoral poets, like Tao Yuanming and Meng Haoran were always popular, Song poems are also highly praised for its graceful and restrained.

From above analysis we can see that romantic charm, connotation, emotion and intelligence in poems are main creative components, when study this kind of literature works, researchers also shall be with good sensitivity, sensitive observation and penetration on life, thus can understand the substance and artistic conception of poems ${ }^{[3]}$.

\section{Research on novel and drama.}

Except ancient poems cannot leave researchers' sensitivity, ancient novel and drama also need researchers' sensitivity as study basis. Sensitivity for novel and drama is different from sensitivity for poems, this is mainly because that novels and dramas contain large number of character relations and story lines, therefore, this kind of sensitivity needs to judge and speculate character images and character's destiny, that is to restore literature works back to life from the logic angle. During the living process of novels and dramas, because of people's different life experience, view of life and world view, the restoring works also need vivid personal colors. For example, in the researches on Chinese ancient novel Romance of the Three Kingdoms, different people have different opinions on the main characters. Most of readers think Liu Bei as a good emperor, Cao Cao is a reversed arch-careerist, Zhu Geliang is the embodiment of wisdom and loyalty. While in Lu Xun's opinion, he 
saw hypocrisy from Liu Bei's clemency, saw straightforward from Caocao's duplicity, saw crafty from Zhu Geliang's wisdom. Characters images' diversity and complexity closely relate to authors' creation, or the author deliberately designed characters' multiple personality, or there is contradiction between authors' description and the real needs. While researchers' understanding on characters' multiple personality cannot leave their perception on the literature works, to summarize this kind of perception to get conclusion.

\section{Historical research on ancient literature.}

Research on the history of literature accounts for most of the Chinese ancient literature researches, historical literature cannot leave historical data record. Among the historical researches on literature works, the creation era, authors' basic situation and features of contemporary literature are all the scope of literature researches. While the research process will be influenced by many limitations, mainly because the creation era was long ago, historical record was not clear or lack of historical record would cause limitations to literature restoring. While in order to get their research purpose, historical researchers will try their best to restore the history and literature. The restoring needs good sensitivity, perception, imagination and inferential capability on literature works can connect historical data thus to restore history through literature works. To some extent, literature history can be understood as authors' mind travel and emotion experience, while grasping ancient people's psychology and emotion is still difficult, restoring history is more difficult. If researchers have no good sensitivity on emotional experience and psychology, thus restoring authors' spiritual appearance, emotional state or psychological status are very difficult.

\section{Sensitivity cultivation of ancient literature researchers}

\section{Enlarge reading quantity.}

One's sensitivity has a great relation with his//her innate factors, while cultivation is more important. The sensitivity cultivation, first needs to have enough reading quantity, literary attainment is the necessary accomplishment of a qualified ancient literature researcher, while good literary attainment cannot leave reading literature works. Therefore, it can say that literature works reading is a nonnegligible lesson for ancient literature researchers to improve their sensitivity. In ancient people's literary theories, they always thought reading was the main way of literature creation, for example, Du Fu said in his Twenty-two Rhymes for Wei Zuo Cheng Zhang that "Having read more than ten thousand books, writing if there is God". Writing has great relation with research, literature research needs to be carried out on the basis of familiar with literature works, thus can improve students' sensitivity.

\section{Increase life experience.}

In China, literature has always been treated as the study of nature-cultivation, people used to combine readers' acceptance ability of literature with readers' own thoughts, more to give play to their subjectivity, while western pay more attention to objective comparison. As the relation between “Zhiyan” and "Yangqi” stated by Menci, from Menci's statement we can see that people's moral cultivation is directly reflected on his language expression and discrimination ability, also directly affects his personal judgment on literature works ${ }^{[4]}$. In Chinese ancient literature, human being is the main description material, therefore, readers and researchers' study and acceptance on literature directly relates to their life experience, their life experience, ideology, personal belief are parts of life experience, especially life experience accumulation is crucial for improving their literature sensitivity, which affects their academic characteristic. For example, Lu Xun did not see the detachment complacent of seven sages of the bamboo grove from but the miserable of going into the society, he did not get Tao Yuanming's aloof from the world from his rural emotion, but saw his devotion to the mundane, Lu Xun's such kind of perception and analysis has a great relation with his own life experience.

\section{Try literary creation.}

Besides abundant reading quantity and life experience, ancient literature researchers also need to try literary creation to improve literary attainment thus to improve sensitivity effectively. Only through literary creation by themselves can they experience its hardship, thus to experience the 
essence and true meaning of literary creation. As Du Fu said in his poem that "A piece of literature is meant for the millennium, But its ups and downs are known already in the author's heart." meanwhile, for literary critics, they shall have good literary creation ability and aesthetic judgment, thus they are qualified to judge other people's works ${ }^{[5]}$. Though modern literature research has become an independent discipline, literature researchers no need literary creation ability, they study literature works just from their academic training, while from the aspect of literature sensitivity, literary creation experience is indispensable. Through the history of Chinese ancient literature researches, literature researchers in last century were not separated from writers, some famous literature researchers were writer, such as Lu Xun, Zhu Ziqing, Hu Shi, Guo Moruo and so on. This also showed that abundant creation experience has a certain promotion function on Chinese literature research, it can improve researchers' sensitivity and understanding ability. Therefore, modern ancient literature researchers also should try literary creation to improve literature sensitivity to promote their ancient literature research works.

\section{Conclusions}

To sum up, ancient literature research is the main content of Chinese cultural analysis, it is conductive to the heritage and development of Chinese ancient cultures, it is the important pillar of China's construction of socialist spiritual civilization. Except lack of historical records, researchers' low quality level and the insufficient emphasis under the economic benefits, another important reason for the obstacle factors of Chinese ancient literature research is that researchers' low sensitivity, they cannot correctly understand the artistic conception and connotation in ancient literature works, therefore, ancient literature researchers have to perfect their sensitivity through aspects of enlarging reading quantity, enriching life experience and try literary creation to constantly improve their literature sensitivity to lay solid foundation for ancient literature researches.

\section{References}

[1] Su Junsheng. Literature Sensitivity in Ancient Literature Researches, Youthful Days, 2012(16):195.

[2] Liu Qifeng. Ancient Historical Records and Ancient Literature Research, BO Literature: Classics Reading, 2012(10):90-90.

[3] Fang Liping. "Positive” "Negative” "Combination” of Ancient Literature Research, Yuejiang Academic Journal,2015,7(4):108-114.

[4] Zhang Keli. Ancient Historical Records and Ancient Literature Research, Journal of Shandong University (Philosophy and Social Sciences),2011(3):14-24.

[5] Cui Jidong. Discussion on the Literature Problems in Ancient Literature Researches, Beautiful Poems, 2014(13):80-80. 